\title{
Discussion: Significance and Treatment of Clumsiness in Children
}

\author{
Mijna Hadders-Algra ${ }^{1}$ and Albert Gramsbergen ${ }^{2}$ \\ ${ }^{1}$ Department of Neurology and ${ }^{2}$ Department of Medical Physiology, \\ University of Groningen, Groningen, the Netherlands
}

\section{INTRODUCTION}

Mijna Hadders-Algra (Groningen, the Netherlands):

The contributions to this special issue of Neural Plasticity were written by the invited speakers to the international meeting The Clumsy Child: Aetiology, Pathogenesis and Treatment, which was held in Groningen, The Netherlands, from June 6 to 8, 2002. The aim of the meeting was to bring together scientists involved in fundamental aspects of motor development and scientists and clinicians dealing with children with clumsy motor behavior. Two series of sessions dealt with the clinical aspects of children with clumsy behavior, such as their specific motor problems, their co-morbidity, and the prevalence and etiology of clumsy motor behavior. Multiple s€ssions dealt with the role of specific parts of the nervous system in the organization and development of adequate and task-specific motor behavior. These included principles in the recruitment of motor units in order to produce a smooth gradation of muscle force, specific roles of sensory systems such as proprioception and the roles of the motor cortices and the corticospinal tract, the basal ganglia and the cerebellum in the coordination of motor activity. Finally, specific attention was paid to possibilities of treatment and management of children with clumsy motor behavior.

The meeting made it clear that children with clumsy motor behavior constitute a heterogeneous

Reprint requests to: Prof Dr Mijna Hadders-Algra, Developmental Neur(riogy, University Hospital, Hanzeplein 1, 9713 GZ Groningen, The Netherlands; e-mail: m.hadders-algra@med.rug.nl population. For example, some children with clumsy motor behavior suffer from problems in fine motor skills, others from a dysfunction in balance control, and some do suffer from multiple motor problems (Hoare, 1994). The challenge of the Groningen meeting was to bridge the present day knowledge on the physiology of the various parts of the nervous system involved in motor control and clinical knowledge on the child with clumsy behavior.

Meetings like the Groningen Meeting often result in a collection of interesting contributions and, hopefully, the attendant or reader is able to select some useful messages. This type of meeting is, however, only successful when it results in the generation of new ideas and-in turn-in new experiments and novel guidelines for treatment and management. In order to facilitate the achievement of this goal, we scheduled a general discussion at the end of the meeting. The audience was invited to ask questions, either directly or by means of written text. The majority of these questions was dealt with during the general discussion, which was recorded on tape. The remaining questions were distributed among the invited speakers. The answers and the recorded discussion were edited and authorized by the persons involved.

\section{CLUMSY VS.NORMAL MOTOR BEHAVIOR}

Albert Gramsbergen (Groningen, the Netherlands):

During the last three days we have been talking about clumsy motor behavior. But what is 
normal, skillful behavior and when do we talk about clumsiness? Or, should we rather use the concept of optimal versus non-optimal motor behavior ( $c f$. Prechtl, 1980), and then the obvious problem is to define optimal skillfulness. A third point is, how do we diagnose clumsiness, and in relation to that, does a poor performance on an often used test, the Movement ABC (Henderson \& Sugden, 1992) reliably indicate the presence of clumsiness and-vice versa-does an adequate performance on this test exclude the presence of motor problems?

\section{Hans Forssberg (Stockholm, Sweden):}

When talking about 'normality' with regard to skilful behavior, it should be taken into account that the variation in achievement of children of a certain age is very large. For instance, if you test a group of children aged 12 years, some would perform like a child of 7 years and some would do the test like a child of 16 years. So, it is not easy to define the borders of normality. Actually motor performance of a population can be regarded as a panorama of performances ranging from an optimal performance via many forms of normality to the lower extreme of achievement. At the lower end we probably deal with pathology, i.e., motor behavior not belonging to the repertoire of the intact nervous system. It is also important to keep in mind that we deal with individual end-points of motor development. Not everybody is endowed to become a piano concert player. Thus, also healthy adults show a large inter-individual variation in motor performance.

\section{Brian Hopkins (Lancaster, UK):}

The issue of 'what is normality' is not new and as such has stretched many minds in the past. One of the best treatments of the concept of normality that I have come across was done by an epidemiologist called Feinstein (1977). Feinstein wrote that essentially epidemiologists deal with two sorts of normality: isolated normality and correlated normality. When you simplify it, you could say that isolated normality is derived from the performance and the norms of a single standardized test. Correlated normality on the other hand relates to the performance on sets of tests. If a child scores high on one test of motor performance, that child should also score high on another test of motor performance. Now, the problem with both forms of normality is that there is no utopian ideal of normality.

Thus, what constitutes normality in one context or in one culture is going to differ enormously in another one. For example, if you take something like age at the onset of walking, you expect on average a child in most Western European countries to do that sometime around the age of 12 to 13 months. This is isolated normality according to Feinstein. But, in another culture where, for example, they swaddle the children for quite a long time during the first year of life, there could be some delay in this onset. I once did a study that involved mothers in Jamaica and mothers from England and India (Hopkins \& Westra, 1988). What I came across to my great surprise was that the Jamaican mothers, and also to some extent the Indian mothers, had a specific system of handling the babies. They massaged and stretched the babies. Furthermore, they gave them active exercises to promote two things. The first one was to get them sitting as early as possiblethis was mainly the aim of the Jamaican mothersthe second one was to make sure that the infants could walk as early as possible. These exercises are quite rigorous and when you show them to English or Dutch mothers, they are incredibly shocked by it. But these exercises do have an effect. So, the children who get this form of 
handling sit and walk earlier than the children who do not, even if they belong to the same culture.

Another aspect of normality is what the parents, not clinicians, view as normal. For instance, for the Jamaican parents, it was not really essential that the infant was sitting as early as possible. What was valued in that culture is that when you are at the table eating with the family or friends you are able to sit up with your head and back straight and look as though you are being attentive. Thus, that was the ideal child which they were trying to cultivate. Another aspect of that culture was that they did not allow children to crawl. Everything possible was done to prevent the children crawling. Consequently, those children who had got the handling went from sitting to walking and missed out the crawling stage. In the light of the often postulated notion of the universality of developmental sequences, these children could be regarded as abnormal... In-short, we have to look at normality in these two ways: isolated and correlated. But bear in mind that there are enormous cultural differences.

\section{Milivoj Velickovic Perat (Ljubljana, Slovenia):}

Returning to the question 'Which motor behavior is normal and which is not', I think that everybody is able to distinguish the difference intuitively by so-called Gestalt perception. For example, everybody appreciates the movements of a ballet dancer and everybody dislikes the movements of persons with spasticity and the movements during epileptic seizures. I believe that this Gestalt perception is a sort of innate capacity.

\section{Manuel Hulliger (Calgary, Canada):}

I would like to make a comment related to what Dr Hopkins said on correlated normality.
This is based on some experience with the opposite end of a similar complex problem, namely what is the definition of abnormality. In particular, I have experience in the identification of abnormal versus normal gait patterns. There are certain similarities-in terms of complexitybetween gait analysis and the identification of clumsy, not so skilled, or skilled movements. Common to both is that we are looking at highly multidimensional phenomena. What a clinician or just an attentive observer tends to do is to take in a multidimensional view of motor behavior. For example, a clinician typically will say, when a patient walks in, "This person has had stroke," because there are certain constellations of features which immediately catch the eye. The term Gestalt has been used, which goes in that direction. It is a describing term for multidimensional phenomena. What I think is important, when we try to define normality in a multidimensional space-you can imagine $n+1$ dimensions, each measuring certain parameters of motor performance-is that there appears to be an element of tolerance. That is, we still accept something as normal (normal in a multidimensional space) when it belongs to a cluster of behavior. Within the cluster of normal behavior, there is a tolerance for deviations either in the one direction or the other. Everybody can come up with examples, that we know people who appear to be perfectly normal in their motor behavior, but who have very strange handwriting. Or when they have to tie a knot it takes them five times longer than other people. Still you don't call these persons unskilled. Thus, there seems a kind of weighted constraint on deviations, so that individual deviations do not immediately strike us as being abnormal. This is my intuitive description of the way in which we are in fact rating normality. If we focus on a single parameter and ignore the tolerance for deviations in multiple directions, we-possibly erroneously-would classify persons as being pathological in certain skills. 


\section{Richard Ivry (Berkeley, USA):}

I want to follow up on the comment that we could just look at motor behavior and see whether it is normal or not. Such a belief might be biased by a perceptual system that seems to categorize phenomena either as normal or abnormal. Our conceptual system seems to like such divisions. We seem to have a huge tolerance for variation in the sense that most things look OK. Yet other things stand out and appear to be abnormal. But when you measure the motor phenomena, you are likely to discover that things are much more continuous. Thus we have a conflict here: a mind that prefers to divide things up into tidy categories versus actual measurements that suggest much more continuity in performance variation.

\section{Brian Hopkins (Lancaster, UK):}

I wanted to comment on what Dr Ivry just has said. Human beings do tend to categorize along continua. I think-and presumably this is what $\mathrm{Dr}$ Velickovic meant with his remark on perceiving by Gestalt perception - there will be no disagreement on what constitutes a normal movement pattern and what constitutes the other extreme, an abnormal movement pattern. But because we categorize, we create a grey area in the middle of the continuum-an area which we could call 'mildly abnormal'. However, in general we really don't know what this category means. It is a sort of catch-call category. I think this is the problem with which we are dealing when we are talking about clumsy children. Is it a catch-all category?

Tatjana Velickovic (Ljubljana, Slovenia):

After all these days I would like to raise a question to all of us: is clumsiness a diagnosis, is it a symptom, or is it a syndrome?
Lex Kalverboer (Groningen, the Netherlands):

I am missing in this discussion the concept of optimality, a concept which was very much cherished in the Groningen area (Prechtl, 1980). Now I don't think that this concept is still as flourishing as it once was. But I would be interested in your view on it. The basic idea of the concept was that it would be easier to distinguish between optimal and non-optimal than between normal and abnormal. What is the present status of the optimality concept?

\section{Richard Ivry (Berkeley, USA):}

The problem of the term 'optimality' seems to me that it might be impossible to define an 'optimal' level of coordination or skillfulness. I never heard of somebody being too coordinated and I can't think of what sort of problem you would face if you were too coordinated. In other domains, this might be possible; for example, height. You probably can be too tall, perhaps because of a strain on the vascular system. Probably there the midpoint is optimal in terms of bioenergetics. I have the feeling that with coordination we have the same type of normal distribution, and it might be possible to ask if the mean was optimal. But I don't think this would be reasonable, especially since it would suggest the potential of being 'too coordinated'. That is what I would associate with optimality and why I don't think it useful for understanding variation in coordination.

Brian Hopkins (Lancaster, UK):

The question on the concept of optimality is a difficult one. The idea behind the optimality concept as developed in Groningen is that you more clearly can pinpoint what is optimal in pregnancy or 
in newborn neurological responses than what is normal. Optimality was used in two ways in Groningen (Touwen et al., 1980). It was used with obstetrical data, where obstetrical optimality indicates how optimal the pregnancy was, or in other words how healthy it was. And it was used as neurological optimality in the newborn neurological evaluation. The neonatal neurological optimality score consists of a set of responses or behaviors and for each item the optimal range was defined as the age-appropriate norm of healthy full-term infants. Adding up the number of items with an optimal performance results in an optimality score. What you don't have in this score is a clear cut distinction between optimality and non-optimality. You get optimality and reduced optimality. The difference is a little bit more than a question of semantics. The problem I had with this way of dealing with the functional integrity of the young nervous system is that all items have the same weighting, although one item might be more important than another. A further disadvantage of the use of optimality scores (but one that can be dealt with potentially) is that ideas in obstetrics and about neurological development continuously change. Due, for example, $\omega$ micro-analytical studies on motor development the landscape of what constitutes optimal and normal development is changing. Therefore, the list of items making up the optimality score and the criteria for optimality must change as well. The same holds true for obstetrical optimality. New discoveries or changed insights should be implemented in the list of optimality. I used the optimality concept about 10-15 years ago, and even then it was becoming outdated, in particular with regard to neurological optimality.

Lex Kalverboer (Groningen, the Netherlands):

I sha:- your reservations with respect to the use of the optimality concept. But I think its application went further than just newborns and obstetrics. It went also into the work of Touwen on the neurological condition of older children, children with clumsiness. In my point of view there might be a use of the concept, because normality-abnormality is as problematic as optimality-non-optimality.

Mijna Hadders-Algra (Groningen, the Netherlands):

Maybe I could add to that that in the material of the Groningen Perinatal Project (see HaddersAlgra, 2003-this issue) we tried to use the optimality approach also in the older children. It led us nowhere. The approach of clinical clusters of neurological dysfunction, such as the presence of clear choreiform dyskinesia or coordination problems, was much more rewarding. When we used the clinical clusters we were able to demonstrate relationships between pre- and perinatal condition and neurological condition at school age, and associations between neurological condition and behavioral problems. Thus, the approach of clinical clusters of dysfunction worked much better than the optimality approach.

Heleen Reinders-Messelink (Beetsterzwaag, the Netherlands):

When talking about children with clumsy motor behavior, we also might consider the aspect of need of help. Clumsy children who are referred to a clinician in general need help. Maybe the aspect of special needs can add something to the discussion on normality and abnormality of motor behavior.

Mijna Hadders-Algra (Groningen, the Netherlands):

Thank you for this helpful comment. Still, the need of help also depends on how critical the 
environment, the family, and society, are. When the environment is very critical, many children will be in need of help. In other words, we still deal with the issue of how tolerant we are. With the issue as to what we do consider as normal.

Marian Jongmans (Utrecht, the Netherlands):

I am favoring a dimensional approach for the identification of clumsiness - an approach which is also used in the literature on Attention Deficit and Hyperactivity Disorder (ADHD). According to the criteria of DSM-IV, a child needs to exhibit six signs before a specific behavioral trait of ADHD is scored to be present. But, Dr Gillberg told us the other day that he and his team had been using five signs as a cut-off because in their clinical practice that was useful (Kadesjö \& Gillberg, 1998). I have also been doing this and I know Dr. Hadders-Algra applied the same strategy. She made a parental questionnaire out of the criteria of the DSM-IV and worked with the resulting dimensional rating of ADHD (Hadders-Algra \& Groothuis, 1999). In a similar way we could construct a dimensional rating of DCD. I think we really need such a dimensional approach. For I agree with Dr Forssberg that there is a continuum from 'normal' via DCD to $C P$. And I think that we need studies like those of Forssberg and his group, to show us whether clumsiness fits to a dimensional approach or not.

Reint Geuze (Groningen, the Netherlands):

At this point, and also with respect to the questions raised by Dr. Gramsbergen, I think there is a preceding question: What would be a proper taxonomy of the perceptual-motor domain? The Movement ABC taps a few of the skills of our normal motor repertoire and has norms for these skills. But there are so many other skills-for instance handwriting. Handwriting seems to be a function which develops by training and it seems to be a sensitive marker for abnormality in the perceptual-motor domain. So, my question is: what other functions in the perceptual-motor domain are we missing because we do not pay attention to them? Where is the taxonomy of the motor domain? I have not seen a single study addressing this issue.

Ian Whishaw (Lethbridge, Canada):

I think that you are quite right in pointing out that there is not a motor taxonomy. But in the animal literature people are starting to develop one. There are certain principles on which the nervous system seems to develop. For example, you can identify mechanisms for posture, you can identify that the mechanisms for movements are different from those involved in the maintenance of posture. There certainly is a rich literature on this question (Golani, 1992; Milner \& Goodale, 1995).

\section{Richard Ivry (Berkeley, USA)}

I would like to follow-up on Dr Jongmans' comment about normal versus abnormal versus pathological motility. I was struck by the fact that in almost every paper presented during this meeting people said "We excluded people with CP" implying that $\mathrm{CP}$ is a special case. This seems to be at odds with the idea of one big continuum. I do not know whether we deal with a continuum or not. But when people consistently say that they excluded people with $\mathrm{CP}$ or excluded people with a neurological disorder, the implication is that the included cases do NOT have some underlying neurological correlate of the motor problems.

Saskia Vriesinga (Lelystad, the Netherlands):

I am wondering whether $\mathrm{CP}$ should be included into the spectrum. In pediatric physiotherapy we treat children who are clumsy. Occasionally children get their medical work-up first after referral 
for treatment. It then can happen that the MRI scan of the brain reveals a brain lesion and that the child is diagnosed as having CP. This type of experience favors the idea of a continuum. Sometimes children with clumsy motor behavior are classified as having $\mathrm{CP}$ and others are not.

\section{Hans Forssberg (Stockholm, Sweden):}

If we now enter the issue of $\mathrm{CP}$, it is getting just as messy because also $\mathrm{CP}$ is not a single diagnosis but an umbrella term covering many different motor disorders (Mutch et al., 1992). Basically, the motor dysfunctions of $\mathrm{CP}$ can be divided into two categories, i.e. into positive and negative symptoms (Forssberg \& Hadders-Algra, 2002). The positive symptoms consist of pathological features added to the motor behavior. These are the dysfunctions well known to clinicians, such as spasticity or the persistence of so-called developmental reactions such as the Moro reaction. These types of behavior are abnormal. Negative symptoms are paresis and central dyscoordination. The central dyscoordination stands for a deficient neural control and reflects a failure to develop proper sensorimotor mechanisms. In our research we especially focus on the latter, that is the sensory-motor integration. This is a function where you probably will find a dimension. Thus, I think, that in some aspects of motor behavior you will have dimensions which probably will include both children with $\mathrm{CP}$ and children with clumsy motor behavior. This might hold true for central dyscoordination, but not for spasticity.

Mijna Hadders-Algra (Groningen, the Netherlands):

Thank you for your comment. If we now summarize what has been said, we probably could say that we have a normal distribution of motor abilities, with some persons moving very skillful, a lot executing movements less skillfully, and at the lower tail of the distribution children who for unknown reasons perform less well. Part of the latter group probably has some kind of pathology.

\section{SPECIFIC SUBPOPULATIONS OF CLUMSY CHILDREN}

This brings us to an issue raised by one of the participants. Clumsiness sometimes is associated with marked hypotonia. The question is how to deal with hypotonia. Does hypotonia constitute a form of pathology or is it one of the normal variations? In addition, should children with hypotonia be considered as a specific subpopulation of the children with clumsy motor behavior? Could Dr. Mercuri comment on this?

\section{Eugenio Mercuri (London, UK):}

Since I started to work with children with neuromuscular disorders, I have seen many clumsy children with hypotonia who are referred to investigate whether they have a neuromuscular disorder. Many of these children don't have a neuromuscular disorder and have no muscle weakness, but just show hypotonia and distal laxity. Their motor problems are mainly related to balance but they often do not have serious problems in manual dexterity. The profile of these children is different from that of other children with clumsiness who usually have more problems in manual dexterity and ball skills. So, probably children with hypotonia and distal laxity form a special subgroup within the population of children with clumsy motor behavior.

Mijna Hadders-Algra (Groningen, the Netherlands):

At present, in many countries the Movement $\mathrm{ABC}$ is used to classify children as having clumsy 
motor behavior. But as Dr. Geuze already pointed out, by doing so we often miss the children with a bad handwriting, the dysgraphic children. How should we deal with this problem? What is your opinion Dr. Schoemaker?

Marina Schoemaker (Groningen, the Netherlands):

In the Netherlands several meetings involving all rehabilitation centers were organized to explore the possibilities to reach consensus on the clinical criteria for DCD. We evaluated performance of a sample of clinical referrals with clumsy motor behavior. Eighty percent of the sample could be classified as DCD as they scored below the $15^{\text {th }}$ percentile of the Movement $\mathrm{ABC}$. The remaining $20 \%$, however, did not score that low on the Movement $\mathrm{ABC}$, but still they were referred to a rehabilitation center because of motor problems (Reinders-Messelink et al., 2002). Therefore we started a new project to assess motor problems in children referred to a rehabilitation center because of clumsy motor behavior. In this project we use several tests with the aim of finding the optimal combination of tests with which all children with DCD, who are in need of help, can be detected. The battery of tests includes the Movement ABC, a standardized Dutch test on handwriting (BHK; Hamstra-Bletz et al., 1987) and a test on visualmotor integration (VMI; Beery, 1982).

\section{NEURAL MECHANISMS}

Mijna Hadders-Algra (Groningen, the Netherlands):

We move on to another question raised by the audience. What is the significance of the presence of speech- and language problems in children with clumsy motor behavior? Does it indicate that there are problems in the cerebellum or in the prefrontal cortex?
Richard Ivry (Berkeley, USA):

I don't think that we should expect that neural speech systems are distinct from neural systems involved in manual dexterity. In other words, if a person has difficulty in making coordinated hand movements, it is quite likely that the person also would show a deficit in speech production. Both motor functions use many of the same motor mechanisms. There are certainly parts of the brain, like Broca's area or perhaps the more medial part to Broca's area, that seem to play special roles in-for instance-the representation of syntactic productions of language or which are involved in the construction of higher order units of speech. But at a lower level, speech systems are dependent upon the same brain mechanisms which are also involved in producing coordinated action. For example, if a person has a cerebellar lesion, the person may well have similar movement disorders in manual actions and speech. Moreover, in turn these combinations of problems are going to be different from those occurring in patients with lesions of the basal ganglia or posterior parietal cortex.

Ian Whishaw (Lethbridge, Canada):

I do not quite agree with you, Dr. Ivry. For me the biggest discovery about the brain in the last 20 years has been that there are so many parallel systems. You can trace the parallel systems from their receptors all the way to the motor output (cf. Hikosaka et al., 1999). We talked in general terms about the function of the basal ganglia and the frontal cortex, but we know that there are many parallel loops within both of those systems. It is inconceivable to me that each child who gets brain damage in a certain location is going to be the same. It is probably very unlikely that only one subsystem will be damaged. The implications are 
that if you would look very carefully at behavior after such damage, you should see lots of islands of rather good performance and some islands of poor performance. We are just on the threshold of trying to design techniques in order to fractionate the behavior and correlate specific behavioral traits with neural subsystems. So, for me the revolution about the brain recently is that it is not made up of large homogeneous areas, but of many subsystems. Philosophers now have the binding problem: how do the subsystems interact with each other?

\section{Richard Ivry (Berkeley, USA):}

Why then, Dr Whishaw, do you get so much co-morbidity in children with clumsy motor behavior? If I follow you correctly, you would not predict such a correlation across different developmental disorders.

\section{Ian Whishaw (Lethbridge, Canada):}

Well, what you find depends in part what you are looking for. For instance, in the neuropsychology literature it is known that there are people who have problems identifying words that are related to animals, and there are other people who have trouble identifying words that are related to things. But if you are just looking for language disorder you would not distinguish between those two. So, I think a lot of the confusion we have can be brought back to the problems of taxonomy, as mentioned earlier by Dr. Geuze. So perhaps we should investigate in more detail, as details can be very revealing.

\section{Richard Ivry (Berkeley, USA):}

An additional comment - there may be well a difference between an acquired disorder and a developmental disorder. Acquired disorders in general have this great deal of specificity, but this may be different for developmental disorders. Perhaps in developmental disorders we deal with some sort of general variation in terms of brain development: some people have 'noisier' brains than others. The co-morbidity could be due to generalized changes of this sort.

\section{Adele Diamond (Waltham, USA):}

People mentioned during the symposium already that there are co-morbidities with DCD and ADHD, dyslexia, and other language problems (Gillberg, this issue; Henderson, this issue). So, it is known that there is a certain amount of overlap between language problems, motor problems and ADHD. In terms of involvement of the prefrontal cortex, there is still much we don't know. It also should be realized that the cortex is easier to study than the subcortex because it is closer to the surface. It is easier to image it, it is easier to do surgery on it. I think that in the future we may find that some of the functions that we historically have attributed to the cortex may not be cortical. Maybe they are subcortical. For example, in the discussion on ADHD, attention has focused on prefrontal cortex and not on the striatum (cf. Barkley, 1997). On the other hand, you hear about how the dopamine transporter gene and how the D4-receptor gene are involved (Farone \& Biederman, 1998). But those are genes that should affect striatal function more than prefrontal function. So, I think that as we learn more, we may find that the functions we thought were prefrontal are actually subserved by other systems in the brain. It must be realized that prefrontal cortex can't do everything. At present there are an enormous number of functions that are attributed to prefrontal cortex.

Ann O'hare (Edinburgh, UK):

I just wanted to make a comment in relation to cerebellum and speech. Clinically the children 
who have got developmental anomalies of the cerebellum are intriguing, because of their heterogeneity in clinical presentation. Some of those children will simply have delayed speech development, that looks just like a phonological delay. Other children will have a scanning dysarthria that looks almost like an acquired disorder, while others will have a marked speech production problem, like a verbal dyspraxia. And still all those children do show similar abnormalities at imaging.

\section{Richard Ivry (Berkeley, USA):}

This heterogeneity is also known in the literature on people with stroke or cerebellar degeneration. The cerebellar speech dysarthrias are associated with lesions within certain regions of the cerebellum. We probably once again deal with a looped structure as Dr. Whishaw mentioned for the basal ganglia. A structure like the cerebellum is likely doing a fairly uniform computation. But different areas of the cerebellum are connected with different target areas and so the computation may be used in very different ways. One region might be linked with areas involved in articulatory gestures; another area might be hooked up with areas that are more involved with the postural system. In this way seemingly similar lesions might induce a great heterogeneity in clinical expression even if the underlying computational deficit was similar.

\section{EARLY DIAGNOSIS OF CLUMSINESS}

\section{Albert Gramsbergen (Groningen, the Netherlands):}

Another problem from the audience is-how do you diagnose clumsiness at an early stage? We do realize that specific motor skills develop at certain ages and the question is whether it is possible to diagnose either developmental delays or abnormalities in motor skills at an early age.

Eugenio Mercuri (London, UK):

The population we see in London consists of children with a high risk for developing motor problems. The tests we do at an early age are the Griffith and other developmental tests. In my opinion, the traditional tests like the global scores of the Bayley and the Griffith scales are not really useful in diagnosing clumsiness at an early age. But maybe, if you would look for individual items of these tests, you might get some useful information. We have recently started to use the 'coins' test of the Movement ABC in children aged 1 $1 \frac{1}{2}$ to 2 years. Although no age-specific norms for performance are available, still, the test gives you an idea of the way the child can pick up the coins, the way he/she puts them into the box, and whether he/she has an idea of spatial relationships. We are also trying to standardize a set of visual-perceptual tasks, which include some simplified items from the manual dexterity scale of the Movement $A B C$ and some other tasks which include the masking of visual perception. But we are just starting and I really do not know whether we will gain from these tasks.

Marian Jongmans (Utrecht, the Netherlands):

The question 'How early can we detect clumsy motor behavior in children' is very valid. A year ago, I started to work in a follow-up clinic for children who have experienced perinatal problems. Many times it struck me that parents are very good describers of their child's development. As researchers we perhaps should try to zoom in onto these parental descriptions and turn them into neat experiments or neat observations or questionnaires so that we can transfer the parents' knowledge into proper standardized assessments. 
Adele Diamond (Waltham, USA):

I would like to say something about early assessment. People tend not to assess infants, but I think now we know enough, so that infant assessment could begin to be possible. We now are able to tell if a motor behavior in infants is normal or not. For example, if a 9- or 10-month-old infant cannot pick up a freestanding object that is really not normal by that age. Nowadays, there are many details we know about motor development in infancy. For instance, Von Hofsten (1998), who did wonderful studies of infant reaching, demonstrated that infants of 7 months are quite precise in being able to predict where a moving object is going to be and grasp it. So, I think we are going to be more and more able to predict what is pretty much average behavior and what is quite different from average.

Marina Schoemaker (Groningen, the Netherlands):

I think we have to take into account the large variability in motor behavior of younger and older children (see for example, Hadders-Algra, 2000). This variability interferes with the detection of mild deviations of the norm. The difficulties in early diagnosis can be illustrated with the findings of the study of Silva \& Ross (1980). They diagnosed 3-year-old children as having DCD. When the children were retested two years later, about $90 \%$ of the children had become normal. One of the explanations is that the variability in the children's motor performance erroneously led to the early diagnosis of clumsiness.

\section{An Oostra (Gent, Belgium):}

As a child neurologist I often see very young children. I agree with Dr Diamond that there are several signs at early ages that can be considered as signs of a risk for clumsiness. It seems even easier to detect them in the first year of life than in the second or third year of life. Examples of such risk signs are hypotonia, difficulties in movement transfer, hyperextension behavior, retraction of the arms in the shoulder-girdle, poor midline organization, or poor coordination while grasping, all in the absence of major neurological signs. I think that we can even predict better at 4 months and 6 months than at older ages whether a child will be at risk for clumsiness.

\section{Ian Whishaw (Lethbridge, Canada):}

I just wanted to make a comment on the early diagnosis and mention the work of Teitelbaum and his colleagues (1998). They asked parents of children with autism whether they had home videos of their children when they were infants. They analyzed these videos together with videos of normally developing infants and reported that motor behavior between the groups differed substantially. So, I think we have really an amazing technology to go on and bridge first diagnosis back to early signs.

\section{Eugenio Mercuri (London, UK):}

There are indeed many symptoms and signs you can see in young children. But the problem is 'why do you see the children?' You have to keep in mind that we deal with two different populations, populations of infants at risk for developmental disorders and populations not at risk. In the at risk population, several items from the Griffith scales or another commonly used developmental tests are useful. The way in which children put the lid on the box is already in the first year of life indicative of whether they will develop a motor problem or not. But here we are talking about a population where you are expecting there might be a problem. So, I am not negating that you can see things early but the problem is 'how early can you diagnose a clumsy 
child when you are not expecting to find clumsiness?' Thus, I think we still do not know very much on the early signs of clumsiness in children who do not have risk factors.

Mijna Hadders-Algra (Groningen, the Netherlands):

I agree with Dr. Mercuri. It will be difficult to detect during infancy children who will develop clumsy motor behavior. In populations of children at risk, we will be able to predict later clumsiness to some extent. But the large majority of children who have clumsy motor behavior at school age are not stemming from an at risk population. In Groningen we have a tradition in looking at infant motor behavior and seeing whether we can find abnormalities. From this research we know that it is still quite difficult to predict clumsy motor behavior. You can predict clumsiness at later age to some extent, but you cannot identify all the children (Hadders-Algra \& Groothuis, 1999; Hadders-Algra, 2003 - this issue). And quite some children who have abnormal motor behavior at early age do not end up as a clumsy child. So, it is not so easy.

\section{INTERVENTION IN CHILDREN WITH CLUMSINESS}

This brings us to another issue. Why should we wish to spot at an early age children at high risk for clumsy motor behavior at a later age? We do want it with the aim of early intervention, but do we really have effective means of early intervention? The literature until now has not been very optimistic (Majnemer, 1998). Who wants to comment on that?

\section{Adele Diamond (Waltham, USA):}

I think that, conceptually, intervention should be possible. Dr Hopkins just told us about the methods of Jamaican mothers that improve their infants' motor function. If these methods work there, they should work here. Also Dr. Forssberg told us that training might work. There is a fair bit of evidence that challenging training can help improve skillfulness and I think it can help in many different domains.

Mijna Hadders-Algra (Groningen, the Netherlands):

I could not agree more with that and we should devise the effective treatments. In addition, we should try to coach parents as best as we can. This also means that we should provide the parents with realistic expectations on the child's motor development by means of an appropriate goal setting. Are there means to achieve this in pediatric rehabilitation?

Anke Meester-Delver (Amsterdam, the Netherlands):

Goal setting in pediatric rehabilitation is a three-step procedure. The first step consists of the provision of extensive information on the child's actual physical and developmental condition, including the functional and behavioral conesquences of these conditions. The information forms the basis for the dialogue with the parents. It is necessary to check whether the information is understood and whether the parents accept the information. If parents do not accept the information provided by the rehabilitation team, their goals for treatment usually differ from those proposed by the team. The second step is the creation of an inventory of the needs for help of the parents and the child. The inventory serves the formulation of functional goals to achieve in a joint effort of family and team. The third step consists of the construction of so-called discipline goals, i.e. goals which are formulated in a SMART (Specific, Measurable, Acceptable, Reasonable, Timed) way (Roelofsen, 2001). The latter could be 
illustrated with an example. Imagine that the parent's question is that they would like their child to learn to play by himself for a while, so that they can pay more attention to the siblings. The goal at the discipline level of the occupational therapists could then be that the child owns within a period of 6 weeks an adequate chair, that he is able to sit in a comfortable way for a period of 15 minutes, and that he can use his hands with an adequate grasp and release pattern. The goal at the level of the physiotherapist could be that the child's head balance should improve within a period of 6 weeks so that he will be able to hold his head while sitting in his chair while looking and playing with toys for a period of 15 minutes. In addition, the goal for the teacher could be that the seated child in 6 weeks time is able to play attentively with appropriate toys for a period of 15 minutes without needing help.

Mijna Hadders-Algra (Groningen, the Netherlands):

In line with good tradition in science, our meeting on clumsy motor behavior in children has generated more questions than answers-questions which we should address in the near future. Examples of such questions are:

Can specific forms of clumsy motor behavior be distinguished? If so, what are the pathophysiological mechanisms of the various types of clumsy motor behavior? Answers to this question will form the vital basis for the development of effective intervention therapies.

Will it be possible to detect at early age children who have a substantial risk of developing clumsy motor behavior? Detection at early age would provide possibilities of intervention at early age, i.e. at an age during which the brain is characterized by considerable plasticity.

It will not be easy to get the answers to these questions. It will require joint efforts of clinicians and neuroscientists. The type of physiological research requested is rather a strategy focusing on systems behavior than an approach based on molecular biological techniques. Clinical research might benefit from longitudinal, randomized intervention studies using multiple standardized ways to monitor the child's motor behavior and functional performance.

\section{REFERENCES}

American Psychiatric Association. 1994. Diagnostic and statistical manual of mental disorders, $4^{\text {th }}$ edition. Washington, DC, USA: APA; 943.

Barkley RA. 1997. Behavioral inhibition, sustained attention, and executive functions: construction of a unifying theory of ADHD. Psychol Bulletin 121: 65-94.

Beery KE. 1982. Revised administration, scoring, and teaching manual for the developmental test of visual-motor integration, $2^{\text {nd }}$ edition. Toronto, Ontario, Canada: Modern Curriculum Press; 112.

Farone SV, Biederman J. 1998. Neurobiology of Attention-Deficit Hyperactivity Disorder. Biol Psychiatry 44: 951-958.

Feinstein AR. 1977. Clinical Biostatistics. St Louis, Missouri, USA: Mosby; 468.

Forssberg H, Hadders-Algra M. (in press) Pathophysiology of movement disorders in cerebral palsy. In: Velcikovic Perat M, Neville B, eds, Cerebral Palsy. Amsterdam, the Netherlands: Elsevier Science Publ.

Gillberg C, Kadesjö B. 2003. Why bother about clumsiness? The implications of having developmental coordination disorder (DCD). Neural Plast 10: 59-68.

Golani I. 1992. A mobility gradient in the organization of vertebrate movement: The perception of movement through symbolic language. Behav Brain Sci 15: 249-308.

Hadders-Algra M. 2002. The Neuronal Group Selection Theory: an attractive framework to explain variation in normal motor development. Dev Med Child Neurol 42: 566-572.

Hadders-Algra M. 2003. Developmental Coordination Disorder: is clumsy motor behavior caused by a lesion of the brain at early age? Neural Plast 10: $39-50$. 
Hadders-Algra M, Groothuis AMC. 1999. Quality of general movements in infancy is related to the development of neurological dysfunction, attention deficit hyperactivity disorder and aggressive behavior. Dev Med Child Neurol 41: 381-391.

Hamstra-Bletz E, De Bie J, Den Brinker BPLM. 1987. Beknopte beoordelingsmethode voor kinderhandschriften, experimentele versie. Lisse, the Netherlands: Swets \& Zeitlinger; 71.

Henderson SE, Henderson L. 2003. Towards an understanding of Developmental Coordination Disorder: terminology and diagnostic issues. Neural Plast 10: $1-14$.

Henderson SE, Sugden DA. 1992. Movement assessment battery for children. Sidcup, Kent, UK: The Psychological Corporation; 128.

Hikosaka O, Nakahara H, Rand MK, Sakai K, Lu X, Nakamura K, et al. 1999. Parallel neural networks for learning sequential procedures. Trends Neurosci 22: 464-471.

Hoare D. 1994. Subtypes of developmental coordination disorder. Adapted Phys Act Quart 11: 158169.

Hopkins B, Westra T. 1988. Maternal handling and motor development: an intracultural study. Genet Soc Gen Psychol Monogr 114: 377-408.

Kadesjö B, Gillberg C. 1998. Attention-deficits and clumsiness in Swedish 7-year-old children. Dev Med Child Neurol 40: 796-804.

Majnemer A. 1998. Benefits of early intervention for children with developmental disabilities. Sem Pediatr Neurol 5: 62-69.

Milner DA, Goodale MA. 1995. The visual brain in action. New York, NY, USA: Oxford University
Press.

Mutch L, Alberman E, Hagberg B, Kodama K, Velickovic Perat M. 1992. Cerebral palsy epidemiology: where are we now and where are we going? Dev Med Child Neurol 34: 547-555.

Prechtl HFR. 1980. The optimality concept. Early Hum Dev 4: 201-205.

Reinders-Messelink HA, Schoemaker MM, Van der Wees M, de Kloet A. 2002. Description of the characteristics of children treated for Developmental Coordination Disorder (DCD) in rehabilitation clinics. DCD-V Conference "Developmental Coordination Disorder: Mechanisms, measurement, management". Banff, Alberta; 42. (Abstract)

Roelofsen E. 2001. The rehabilitation activities profile for children. PhD-thesis, Free University $\mathrm{OF}$ Amsterdam, ISBN 90-5669-055-8.

Silva PA, Ross B. 1980. Gross motor development and delays in development in early childhood: assessment and significance. J Hum Mov Stud 6: 211-226.

Teitelbaum P, Teitelbaum O, Nye J, Fryman J, Maurer RG. 1998. Movement analysis in infancy may be useful for early diagnosis of autism. Proc Natl Acad Sci USA 95: 13982-13987.

Touwen BCL, Huisjes HJ, Jurgens-Van der Zee AD, Bierman-Van Eendenburg MEC, et al. 1980. Obstetrical condition and neonatal neurological morbidity. An analysis with the help of the optimality concept. Early Hum Dev 4: 207-228.

Von Hofsten C, Vishton P, Spelke ES, Feng Q, Rosander K. 1998. Predictive action in infancy: tracking and reaching for moving objects. Cognition 67: 255-285. 

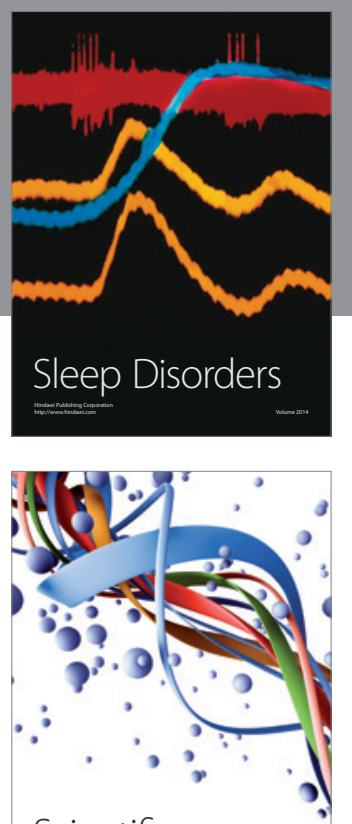

Scientifica
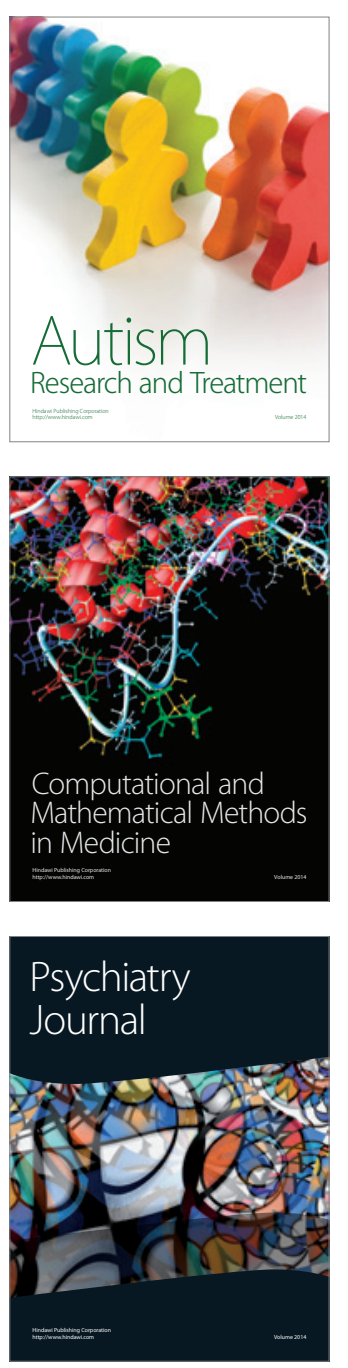
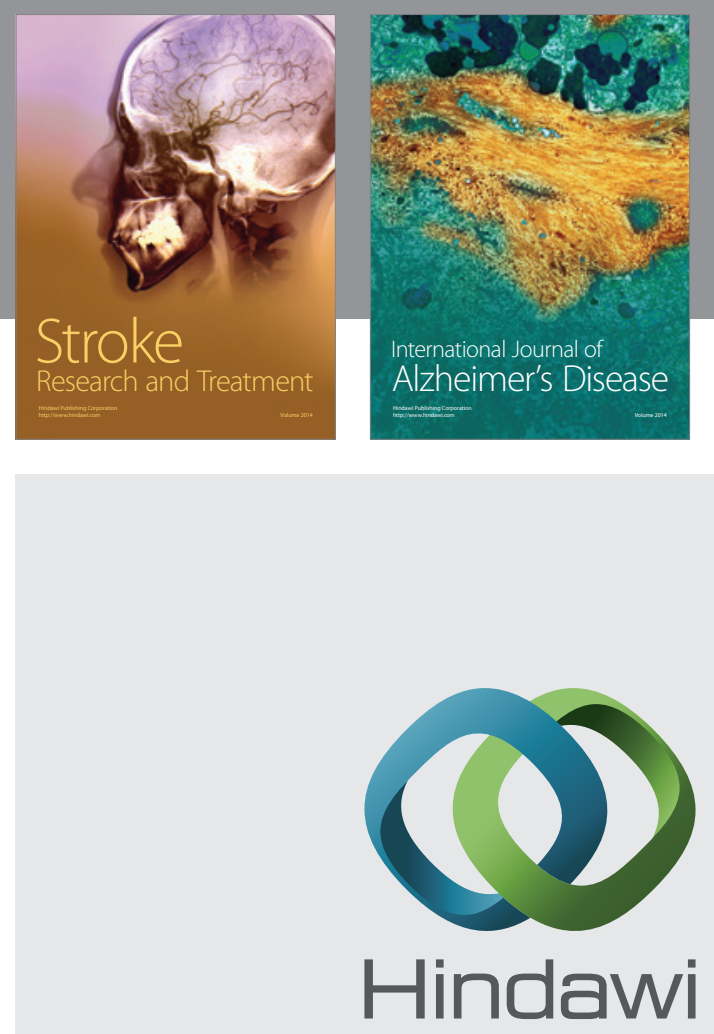

Submit your manuscripts at

http://www.hindawi.com
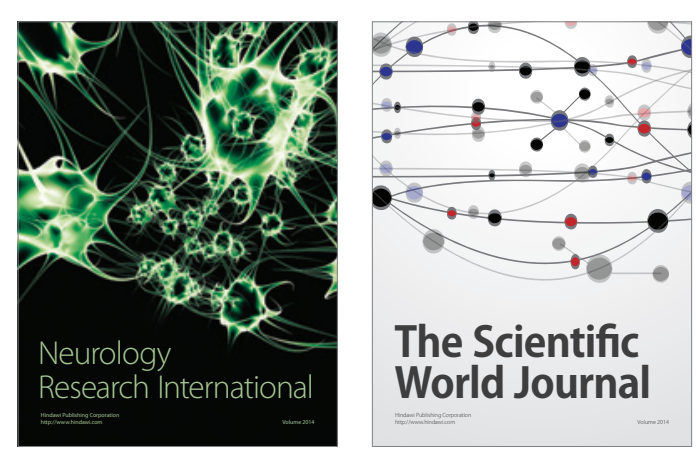

The Scientific World Journal

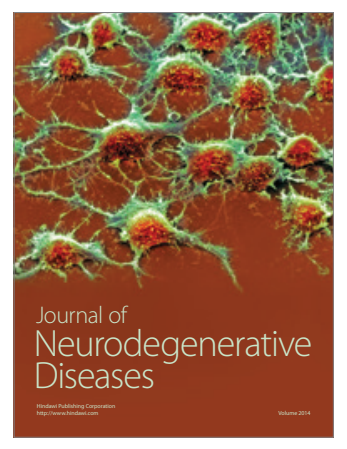

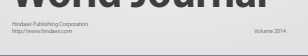

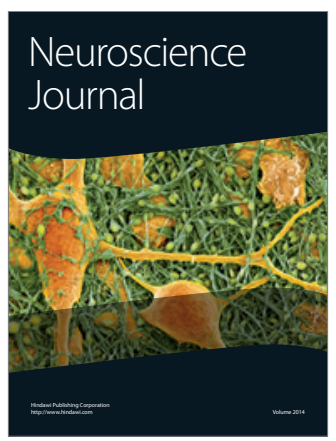

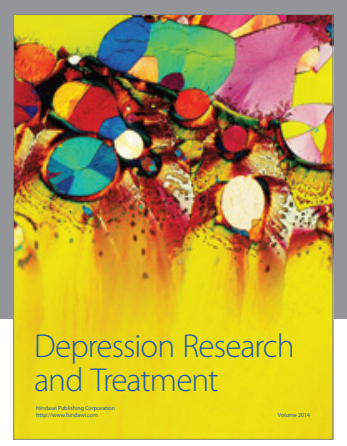
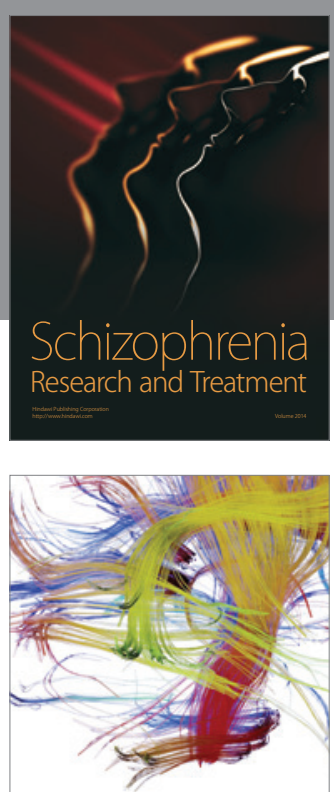

Brain Science

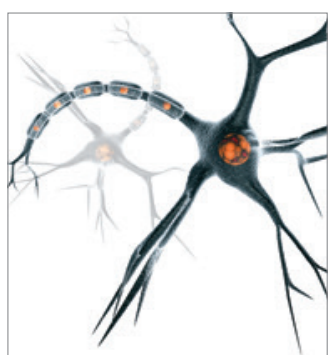

Neural Plasticity
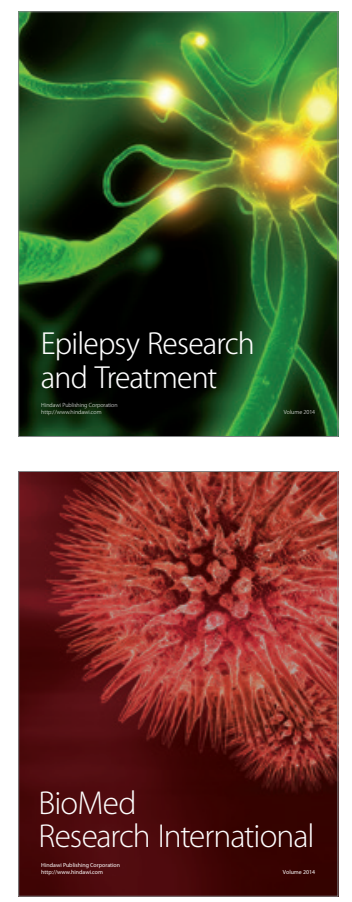

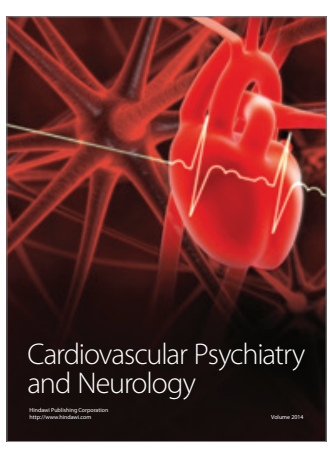

Parkinson's

Disease
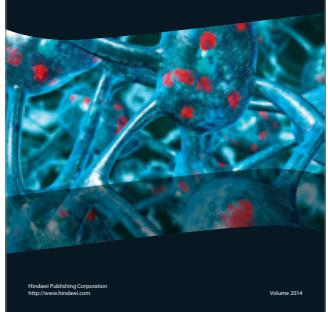